Volume 7, No.2, April - June 2018

International Journal of Computing, Communications and Networking

Available Online at http://www.warse.org/ijecn/static/pdf/file/ijecn46722018.pdf

https://doi.org/10.30534/ijccn/2018/46722018

\title{
ADVANCED IMAGE STEGANOGRAPHY BY MEANS OF SECRET- PIECE NOTICEABLE MOSAIC IMAGES BY NEARLY-REVERSIBLE COLOR TRANSFORMATION
}

\author{
Sujitha.S $\mathbf{S}^{\mathbf{1}}$ Arun Biradar ${ }^{2}$ \\ ${ }^{1}$ Dept. of Computer Science \& Engineering, EWIT, sujithaaa1894@ gmail.com \\ ${ }^{2}$ Head of Dept., Dept. of Computer Science \& Engineering, EWIT, arunbiradar@ewit.edu
}

\begin{abstract}
Another photograph steganography framework is proposed, which makes regularly from a discretionarily picked target picture an expected mystery territory unmistakable mosaic picture as a

surges/undercurrents in the changed over pixels tones, conundrum piece obvious mosaic pictures of high similarities to self-definitively picked target pictures can be made with no need of an objective picture database.
\end{abstract} veil of a given enigma picture. The mosaic picture is yielded by disconnecting the confound picture into portions and changing their shading attributes to be those of the bits of the objective picture. Skilled systems are proposed for use in the shading change process with the target that the mystery picture might be recouped about lossless. The strategy not just has a stenographic impact obliging for secure keeping of conundrum pictures, yet besides gives another approach to manage comprehend the bother of concealing riddle pictures with colossal information volumes into target pictures. Amazing trial happens demonstrate the believability of the proposed strategy. The simulation output is taken by using mat lab software. The algorithm achieving good accuracy and integrity, noise resistance compared to the exiting method. Another image steganography technique has been proposed, which not exclusively can be utilized for secure keeping of perplex pictures yet likewise can be another choice to grasp the bother of masking pictures with goliath information volumes behind cover pictures. By the use of fashioned pixel shading change and moreover able treatment of

Keywords: image steganography, secret image, encryption, decryption.

\section{INTRODUCTION}

Today picture security is an essential issue while transmitting pictures over the web for different applications, for example, for online individual photos, gatherings, private real records, military picture database, storing up frameworks, and remedial imaging structures.

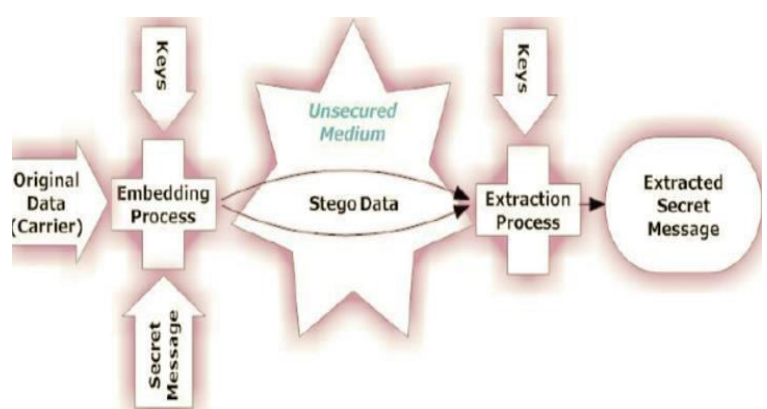

Figure 1: architecture of image steganography

These photographs may contain private or described data with the target that they ought to be shielded from spillages/hardship amidst transmissions. Along these lines different systems have been proposed for 
securing picture transmission, from encryption and information disguising. The basic thought behind the photograph encryption is to transmit the photograph safely finished the system so no unapproved client can have arranged to unscramble the photograph without a confound key. In any case, blended picture will be the purposeless record, which can't give extra data as of now deciphering and may stimulate an attacker's idea amidst transmission process because of its abnormality alive and well. So far in cryptography stores of work have done identified with content data. Encryptionstructures so far utilized for content data may not work same way for visual. Pushed pictures are drawing in information makes with wide use and different clients are hypnotizing to execute content affirmation on them to keep from copyright, review or glitch. On much structure like military picture databases, giving security is must. It is fundamental to shield private picture information from unapproved get to. Encryption is the favored strategy for securing the transmitted information. In any case, number of different frameworks instead of encryption is besides open for changing over imperative piece of data into such shape which get to is restricted to unapproved clients. There are unmistakable encryption structures for encoding and decoding pictures are accessible. In data frameworks, parts of security like game plan, security, affirmation and non-disavowal should be refined. Mosaic is the distinguishing strength of affecting pictures with an amassing of little bits of shaded glass, to stone, or various materials. This control is used here in the region of picture steganography. The new sort of craftsmanship picture called mystery fragment clear mosaic picture contains little bits of a given source picture. Watching such a sort of mosaic picture, one can see every single one of the bits of the source picture, yet the fragments are so little in assess in this way optional in position that the eyewitness can't comprehend what the source picture resembles. The source picture might be said to be unobtrusively inserted in the subsequent mosaic picture, however the fragment pieces are all in all unmistakable to the onlooker. Likewise, this is the motivation driving why the subsequent mosaic picture is named enigma part recognizable. It might be utilized as a transport of a baffle source picture in the camouflage of another, an objective photograph of a substitute substance. It is beneficial for the use of covert correspondence or secure keeping of astound pictures.

\section{RELATED WORKS}

Chin Chen chang, Min- Shian Hwang, and Tung Shou Chen [3] have given a fast encryption algorithm for secure image cryptosystems in 2001. Vector Quantization, cryptography and other theorem is the major platform for the cryptosystems to transmit images. It was a meaningful technique to lower bit rate image compression. In VQ firstly transformation of images into vectors takes place and further vector by vector then are sequentially encoded W. B. Pennebaker tried to explain that the main obstacle in quantity of data required representing a digital image. For this we would have to make image compression standard to maintain quality of the images after compression. To meet all the needs, the JPEG standard for image compression includes two basic methods having different operation modes: A DCT method for "loss" compression and a predictive method for "lossless" compression [4]. I-Jen Lai and Wen-Hsiang Tsai [2] have presented technique for information hiding, which proposes that secret image is divided into tile images and then for mosaic image they were fix to its next target image selected from a 
database. Secret key randomly selects few blocks of mosaic image .

\section{PROPOSED WORK}

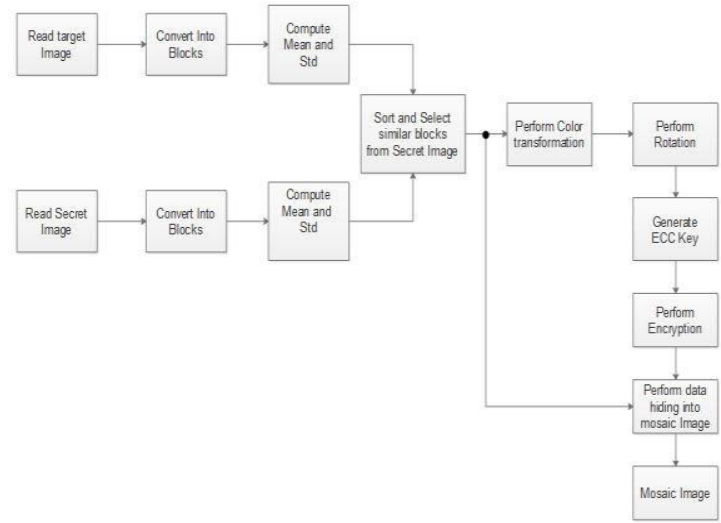

Figure 2: proposed system architecture

In the essential stage, a mosaic picture is yielded, which contains of the parts of an information mystery picture with shading adjustments as exhibited by a similitude perspective in the context of shading arrangements. The stage joins four phases:

- fitting the tile pictures of the baffle picture into the objective squares of a preselected target picture;

- changing the shading common place for each tile picture in the astound picture to twist up that of the relating target dishearten in the objective picture;

- turning each tile picture into a hanging on for the base RMSE respect with yielding to its taking a gander at target piece

- embedding's basic data into the made mosaic picture for future recuperation of the confuse picture.

In the second stage, the inserted data is emptied to recuperate lossless the question picture from the influenced mosaic to picture. The stage joins two phases: clearing the presented data for conundrum picture recuperation from the mosaic picture, and recouping the perplex picture utilizing the removed data.

\section{Algoritham1:}

\section{Mosaic Image Creation and Secret Image Recovery Algorithms}

- This step it is making the mystery part noticeable mosaic picture

- fitting tile pictures into target squares

- divide the mystery picture $\mathrm{s}$ in to number of tiles $\mathrm{n}$. as for $\mathrm{T}$.

- compute the mean and standard deviation of the mystery picture and tiles.

- Sort the quantity of pieces in view of the normal standard deviation

- Create mosaic for mystery picture as for mapping succession $\mathrm{L}$.

- performing shading transformation between the tile pictures and target squares

- rotating the tile pictures embedding the secret image recovery information.

\section{Algorithm 2. Secret image recovery.}

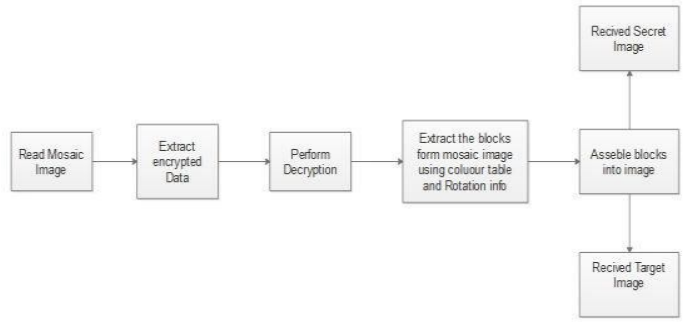

Figure 3 : secret image recovery 
- extracting the mystery picture recuperation data.

- recovering the mystery picture.

\section{RESULT AND ANALYSIS}

The new approach algorithm result as given below

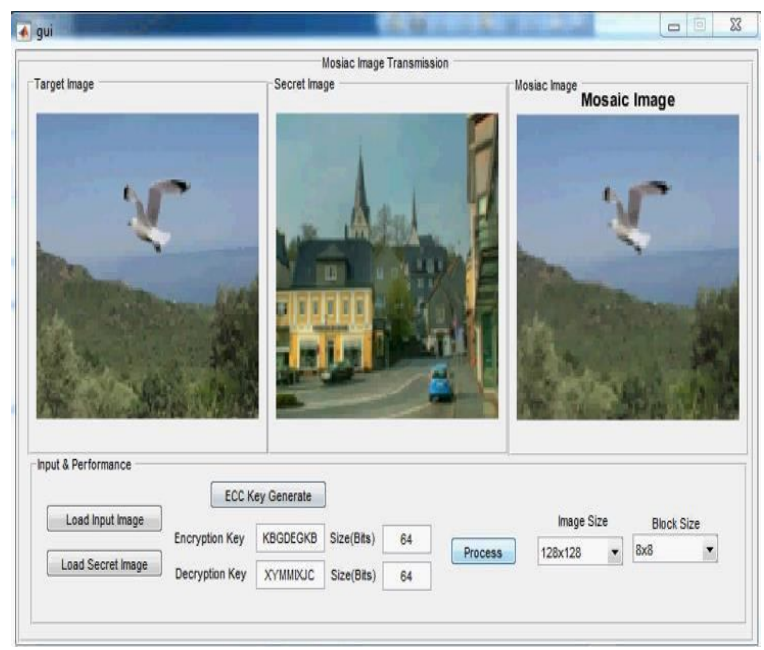

Figure 4 : sending secret image with mosaic (encryption side)

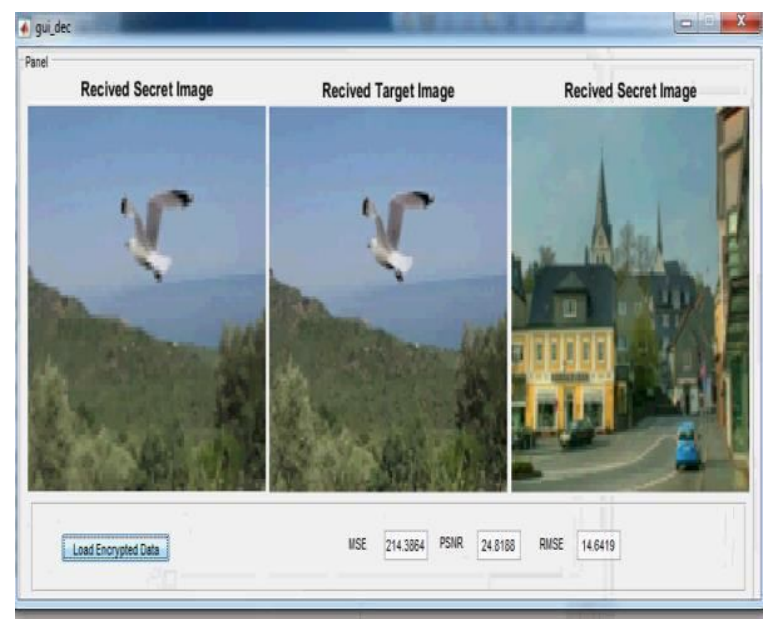

Figure 5 :receiving secret image with mosaic (decryption side)

\section{CONCLUSION}

Another image steganography technique has been proposed, which not exclusively can be utilized for secure keeping of perplex pictures yet likewise can be another choice to grasp the bother of masking pictures with goliath information volumes behind cover pictures. By the use of good 'ol fashioned pixel shading change and moreover able treatment of surges/undercurrents in the changed over pixels' tones, conundrum piece obvious mosaic pictures of high similarities to self-definitively picked target pictures can be made with no need of an objective picture database, and the vital confound pictures can be recouped moderately lossless from the made mosaic pictures. Marvelous test happens have demonstrated the presence of mind of the proposed methodology. Future examinations might be encouraged to applying the proposed philosophy to pictures of shading models other than the RGB.

\section{REFERENCES}

[1] G. Prabhakaran, Dr. R. Bhavani and Kanimozhi, Dual Transform Based Steganography Using Wavelet families and quantifiable Methods."Proceedings of 2013International Conference on Pattern certification Informatics and Mobile Engineering(PRIME) Feb.21-22 IEEE 2016.

[2] StutiGoel, Arun Rana and Manpreet Kaur, "A Review of Comparision Techniques of Image Steganography." Global Journal of Computer Science and Technology, Vol. XIII Issue IV Version I Year 2015.

[3] Ghsemi. E. , Sci. besides, Res. Branch, Islamic Azad Univ., Tehran,Iran, Shanbehzadeh. J.,ZahirAzami. B. "A Steganographic Method Based On Integer Wavelet Transform And Genetic Algorithm."InternationalConferenceOn 
Sujitha.S et al., International Journal of Computing, Communications and Networking, 7(2) April - June 2018, 255-259

Communication And Signal Processing (ICCSP), 2011 IEEE Feb.2013 ISBN: 978-1-4244-97980.

[4] SabyasachiPattnaik, R.K. Chhotaray,K.B.Raja and K.B. Shiva Kumar,"PerformanceComparision Of RobustSteganographyBasedOnMultiples

Transformation Techniques.", International Jouranal On Computer Technology Applications, Vol 2(4), Pp.1035-1047, 2011.

[5] K. B. Raja And H.S. Manjunatha Reddy, "WaveletBasedNonLsbSteganography," International Journal Of Advanced Networking And Applications, Vol 03(3), Pp.1203-1209,2011. General Journal of Computer Applications (0975 - 8887) International Conference on Advances in Science and Technology (ICAST-2014) 34.

[6] Ghosal.N.,Mandal J.K.,"ASteganographic

Schemes For Color Images Authtntication (SSCIA)", International Conference On Recent Trends In Information Technology (ICRTIT 2011). (Madras Institute Of Technology Chennai, India June 1305,2011), IEEE Conference Publications, 826-831.

[7] NilanjanDey, Anamitra B. R, And Sayantan D. " A Novel Approach Of Color Image Hidind Using Rgb Color Planes And DWT, " International Jouranal Of Computer Applications, Volume 36-No. December 2011.

[8] Sarreshtedari.S., GhaemmaghamiS."High

Capacity Image Steganography In Wavelet Domain. In Proceedings Of 2010 seventh IEEE Consumer Communications And Networking Conference (CCNC) (Las Vegas,Nevada, USA, 9-12 January 2010), IEEE

Conference Publications, 1-5.

[9] Chen, R. J., Peng, Y.C., Lin, J. J., Lai, J.L., Horng, S.J., " Novel Multi-Bit Bitwise Adaptive Embedding Algorithms With Minimum Error For
Data Hiding" Fourth International Conference On Network And Systems Security (NSS 2011), (Melbourne, Australia 1-3 September 2010), IEEE Conference Publications, 306-311.

[10] Ali Al-Ataby And Fawzi Al-Naima, "A Modified High Capacity Based On Wavelet Transform,"InternationalArabJournalOf Information Technology, Vol.7,Pp,1-7,2010.

[11] Weiqi Luo, Fangjun Huang And JiwuHuang , "Edge Adaptive Image Steganography Based On LSB Matching Revisited" IEEE Transaction On Information Forensics And Security, Vol. 5, No. 2, June 2011. 